\title{
Cities Talking - A Social Media Platform: Crowdsourcing with Sentiment Analysis and Recommender System
}

\author{
John Kcero Aujero, Nico Manuel Cruz, Jason Joie Padilla, Mary Elysa Pineda, Mark Jezter \\ Ballesteros, Ma. Corazon Fernando Raguro ${ }^{+}$, and Ace Lagman \\ FEU Institute of Technology, Philippines
}

\begin{abstract}
Cities Talking is a crowdsourcing system where users can check-in in local stores (restaurants, food stalls, merchandise stores) located in the Philippines using the geolocation of their device as well as leave comments, stories, and even guides that will be processed under Lexicon Based approach of Sentiment Analysis to determine the polarity (Positive or Negative) rating of the particular location. The system will also have a leaderboard system where users can collect points and badges by checking-in on different places and be ranked up against their friends and other users. The system will also offer a recommendation system to the user where the application suggests near local restaurants, malls, or restrooms, to name a few, based on their proximity and the rating of the place. Since the main essence of the system is crowd sourcing, users can also share the safety level they feel while they are on their pin-location. Their friends can also see the score they inputted as well as the average score of the area based on the total score of all users who checked-in there. To test the effectiveness of the software, the proponents conducted a survey to I.T. specialists; using FURPS model, the result showed a weighted mean of 4.90 which corresponds to excellent.
\end{abstract}

Keywords: crowd sourcing; sentiment analysis; social media; recommender system; geolocation

\section{Introduction}

The very essence of technology today is to improve people's lives, and crowdsourcing is one of the technological era's innovations. According to Evans [1], Most companies understand how critical it is to reach out to consumers and other stakeholders if they want to be successful in the long run. In recent years, an inconsequential but highly effective method of reaching out to others has emerged. Crowdsourcing is a concept that can be found almost everywhere. Weather forecasters and traffic reporters, for example, invite the public to call in and report events, allowing them to cover stories they would otherwise be unable to cover. Journalists and writers often use this strategy to get to the heart of the matter by reaching out to the masses rather than a select few. Crowdsourcing helps businesses work through a fast design process by tapping into the global world of ideas. In order to ensure that your goods or services are perfect, you outsource to big crowds (hence the term "crowdsourcing"). Without a doubt, social media and Crowdsourcing have become one of the most important aspects of our current technological era. These two can truly alter and assist humans in improving their ways of life by developing an application that allows them to share their thoughts and emotions while also evaluating reviews of their dream destination to get a sense of what to anticipate.

This Web and Mobile Application will certainly help the society considering that social media plays a significant role in this technological era. The demand of people from the internet will definitely play a big role to make this project effective, this demand will make way for the crowdsourcing to do his job and give the benefits to the users who are fond of travelling around in the Philippines and give them an idea on what to expect if they go to the places that are being geo-tagged in the web and mobile application. For the researchers, this project will surely enhance their skills and give them valuable insights for their future web and mobile applications. As the technology progresses, we are blessed with wonders we thought would not be possible. You can now shop and order things with just a click away and without needing to leave your home. Navigation is now easy as ever that regular maps became obsolete. You can also now look for top

\footnotetext{
+ Corresponding author. Tel.: + 639154150445

E-mail address:mgfernando@feutech.edu.ph
} 
rated restaurants to places you will go just by searching for it. All these features are achievable because of Global Positioning System or GPS. According to Kyes [2], GPS is a global navigation satellite system that provides location, velocity, and time synchronization. With how common this technology is, it is almost present in every smartphone, cars, or even watches. This feature is utilized by almost all social media platforms either by tagging the location of the picture you just uploaded or finding random strangers to chat near you. GPS has made a huge impact in our society. It has changed the way people communicate and live. GPS has made our environment safer and easier place to live How Has GPS Changed Our Life? [3]. Given that this technology is now prevailing and now accessible to everyone, the proponents of this project would like to take advantage of it and develop a location data platform that can be used to endorse social engagement among users with near proximity as well as encourage agile dissemination of information among places that will serve us a guide or even warning to other people.

\section{Literature}

Based on the work of Jonathan \& Mokbel [4], various essential technologies, e.g. search engines and news media, have been empowered by geotagged data (e.g. photographs or news items). However, the lack of usable geotagged data greatly reduces the effect of such applications. Meanwhile, current geotagging methods rely on the presence of prior information, e.g., reliable training dataset for machine learning techniques. Stella, a crowdsourcing system for image geotagging, is introduced in this article. Stella's high accuracy is achieved by being able to hire employees near the location of the picture, even without knowing its location. Furthermore, Stella also restores its trust in the recorded position to help users understand the accuracy of the result. Experimental evaluation reveals that Stella geotags a picture consistently with an average of $95 \%$ accuracy and $90 \%$ confidence on average.

In another work of Acampora, Anastasio, Risi, Tortora, \& Vitiello [5], TwitterTagger can use the textual content of tweets to geotag them. The tool tags each tweet's content and then conducts two disambiguation to explain the connotations of noun phrases in each tweet and to associate correct locations with each tweet. The goal of this method is to display relevant tweets to a user based on his or her physical location. Even though this approach is linked to Twitter geo-location, it has a different objective than our technique. Indeed, TwitterTagger attempts to geo-tag each individual tweet in order to determine which are important for a given user; however, our approach focuses on geo-localizing a group of associated tweets in order to provide users with the geo-location of events discussed on Twitter.

According to Bhaumik [6], Social networks are an active source for sentiment analysis study as people are always attached to social media like Facebook and Twitter, discussing various topics, promoting business and so on. The sentiment-aware systems are used in business studies, social science analysis and making accurate predictions regarding human behaviour. Several abbreviations are used by users in their social media life as often people want to be quick and thus these terms have become a part of the normal social media vocabulary, like FB for Facebook. Since social networks, especially Twitter, contains individual tweets that are small and many abbreviations which might be difficult to interpret by general Natural Language processing, the VADER library has been used for analysis.

In another work of Desai, et al. [7], Crowdsourcing could be a show in which individuals or organizations get merchandise, administrations, and concepts from a large, open community of web clients. The term, coined in 2006, could be a combination of the words 'crowd' and 'outsourcing', and the method has gotten to be a commonplace marvel within the age of the web. The utility of crowdsourcing through the control of social media has been apparent in emergency observing, crisis arranging and emergency administration, social cohesion and investigate. The wide reach of social-media stages gives get to to the firsthand encounters of 'the many' and grants crowdsourcing and collaboration for the broad mobilization of aptitudes and the creation of systems, administration, and bolster frameworks, which permits problemsolving in record time. Emergency mapping was one of the primary employments of social media in common catastrophes Crowdsourcing has been appeared to be advantageous to fiasco help in numerous nations. For illustration, amid the 2012 and 2013 Colorado rapidly spreading fires. Crowdsourcing played a critical part in centering asset assignment and disaster-relief endeavors to seriously influenced ranges. Crowdsourcing has moreover been utilized to momentarily report wellbeing issues, such as cholera amid the 
2010 seismic tremor in Haiti, and dengue in Thailand and Indonesia. Similarly, critically, crowdsourcing amid restorative crises may moreover help in illustrating infection introduction and modes and designs of transmission.

\section{Methodology}

The data gathering that have been chosen by the developers is the quantitative research. The type of research that have been followed is applied research. In the sampling technique, purposive sampling is used. Under the project development, rapid application development is the one applied to the study.

To have more information about the system, the researchers gathered data by a quantitative approach through a survey that is given out to I.T. professionals or associates. This will evaluate and analyse the suggestions and thoughts of end users that will be included for the betterment of the system. Given the time frame and size of the project, the researchers chose 5 I.T. professionals for them to showcase their project and then ask them to answer the survey instrument using the FURPS model. In conclusion, the method that is used for the data gathering will support and produce additional information that will help the researchers to develop the system.

\section{Project Design}

For the Cities Talking system, the researchers used a microservice architecture that helps create a scalable business model that can easily adapt for newer features. Microservice architecture enables the rapid, frequent, and reliable delivery of large, complex applications. It also enables an organization to evolve its technology stack. As show on the figure below, it depicts the separation of backend and frontend architecture of the system where all the user's web interfaces will first need to send a request in the domain provider server then the server will need it to push a JSON request to the backend server. For the mobile application, it can send JSON request directly to the backend servers. The system has a security feature that will need a JTW token that has expiring date before they can reach on the backend server. If token is valid, it will process the requested API need for the system.

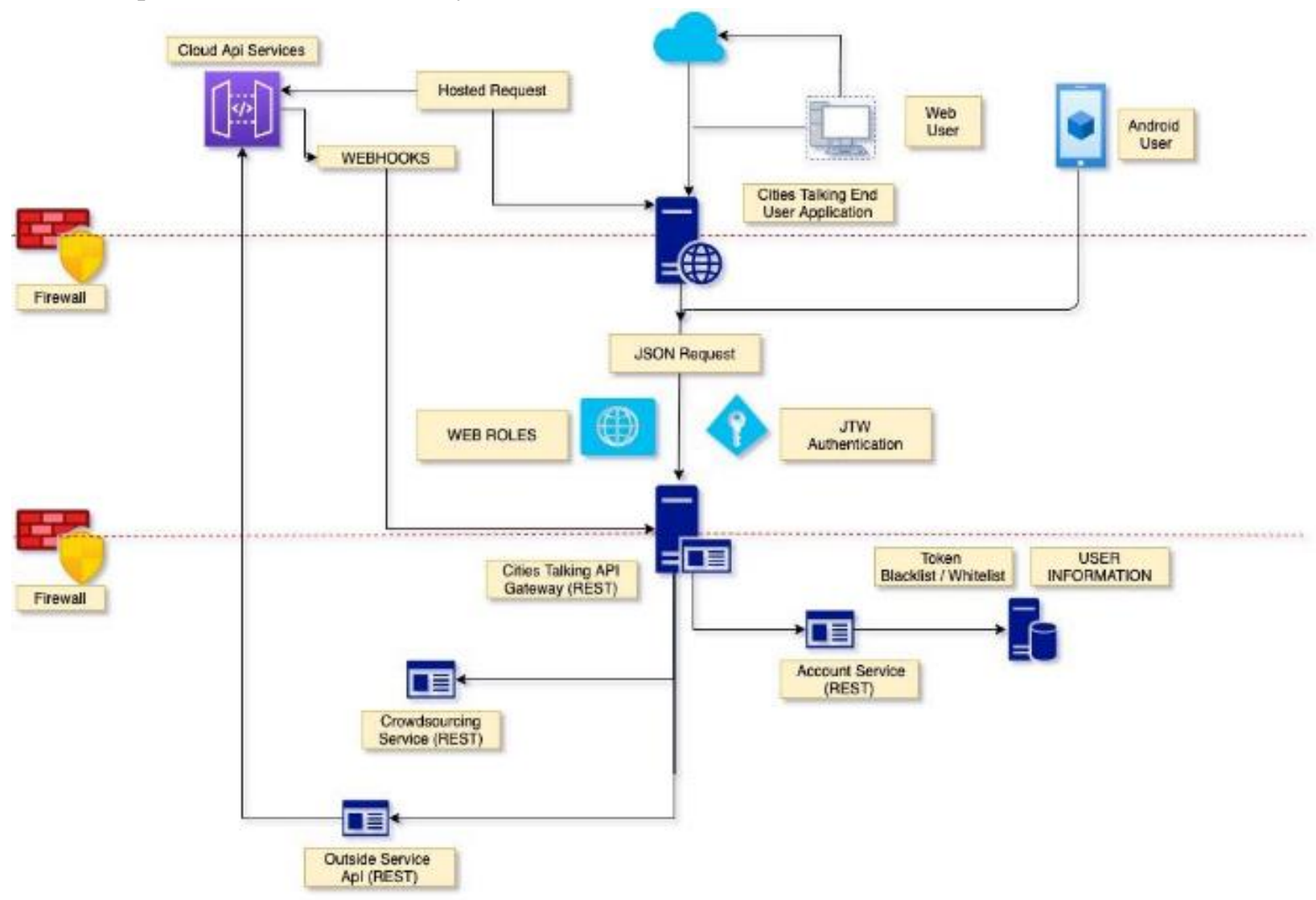

Fig. 1: System Architecture 


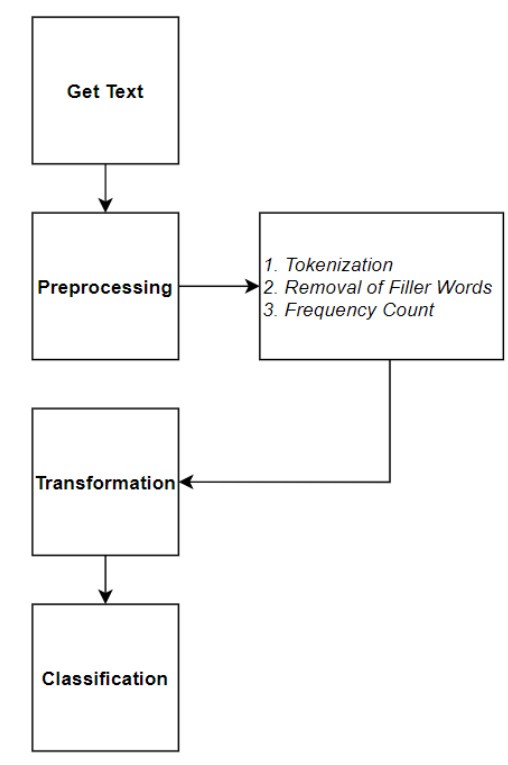

Fig. 2: Process of Sentiment Analysis

According to Gupta [8], Sentiment Analysis is the process of contextual mining of string data to which it identifies and extracts subjective information from the root material. Understanding the user's emotions is the core of the system and people can articulate their feelings and thoughts more willingly than ever before. As such, the researchers chose to utilize the power of Sentiment Analysis to incorporate innovation in aiding human development. There are many types of Sentiment Analysis, from those which detect feelings and emotions, ones that focus on polarity, and models that can identify intentions. For the system, the researchers decided to use the Lexicon-Based Approach where the system identifies the polarity of data input into a positive or negative, statement based on an external dictionary. The semantics then are extracted from the data source and the score is calculated. Figure 2 shows the process of Sentiment Analysis of the system.

For this project, the proponents provided a mobile application prototype using Adobe XD as well as a working admin prototype through web application. The mobile application of the system has three main modules: (1) Talk Module, (2) User Feed, (3) Leaderboard. Figure 3 shows the Talk Module. Here users can share "Talks" regarding the location that they chose. Users can post a comment and then system will analyze it whether if it is positive or negative, then the system will opt different emojis based on the output. Users can select the corresponding emoji they relate to best, and they can also choose the safety measure on the slider provided. Figure 4 shows the page where the users can see their rankings based on their checked-in places and have some friendly competition with their friends. Figure 5 shows where the users can put their reviews, upload photos to their selected place. They can also see the mood, emoji, and the safety measure that they inputted during the talk module. They can also view the average of rating and safety of the areas they checked in.

On the other hand, the admin side of the system can be accessed via the website application of the project. The main admin will be given their credentials then he/she can add more admin once inside the system. The website application of the system has three main modules: (1) Dashboard, (2) Talks, (3) Users. Figure 6 illustrates the admin dashboard on the web application of the system. Visualizations of data are also present here like Pie Charts of the overall talks on the system, Bar Graph of the cities as well data corresponding to the number of positive or negative talks, user visits, and top places. The system also provides Trend Data of the Safety Measure of the cities for the past five months. Whereas Figure 7 shows the View User page where it presents all the information related to a particular user. The admin can see the user's profile data, their total mood inputted, places they have been and its rating. As well as the actual feed of talks of the user. 


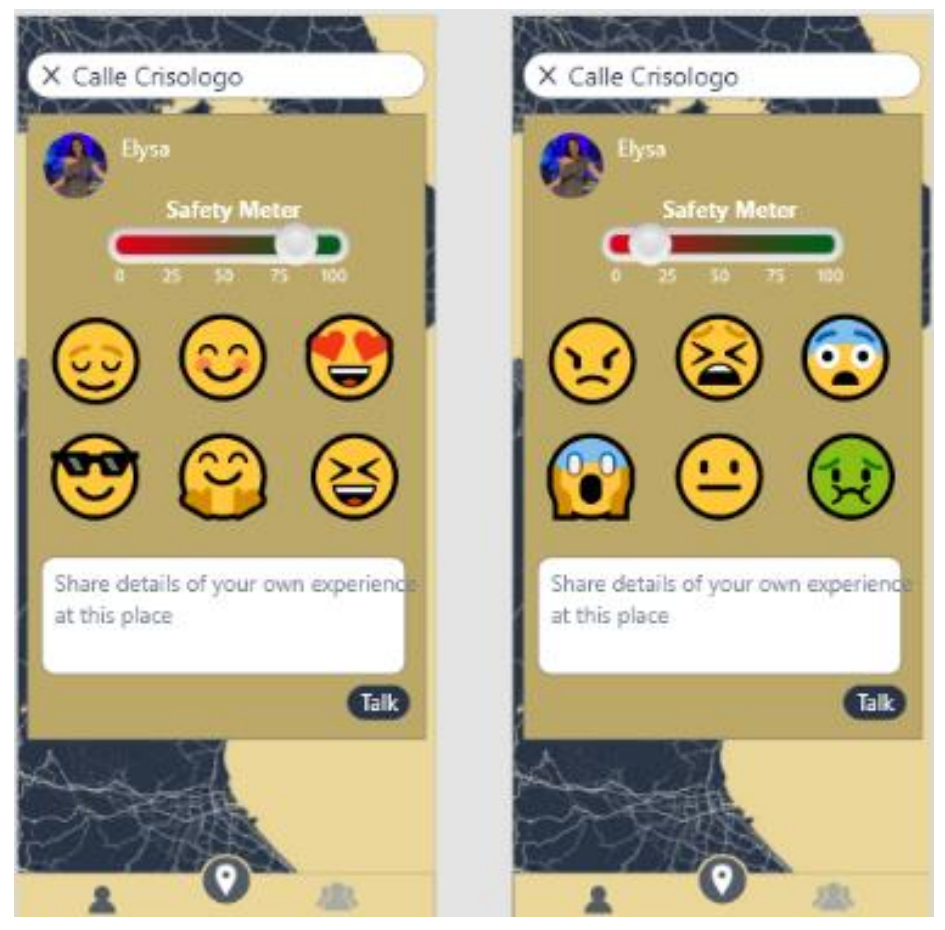

Fig. 3: Talk Module

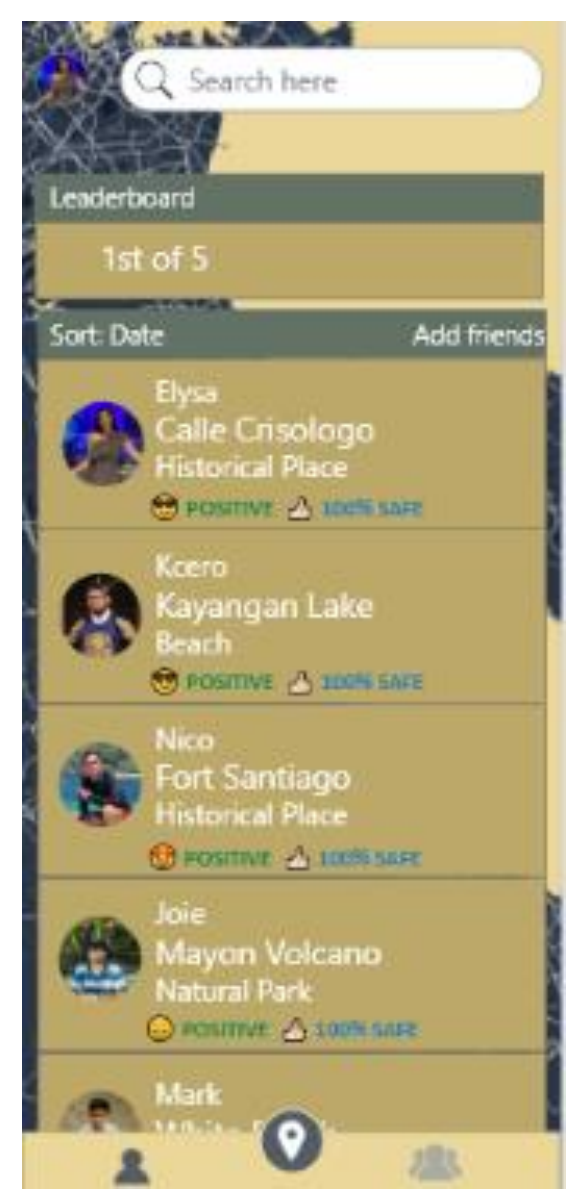

Fig. 4: Leaderboard 


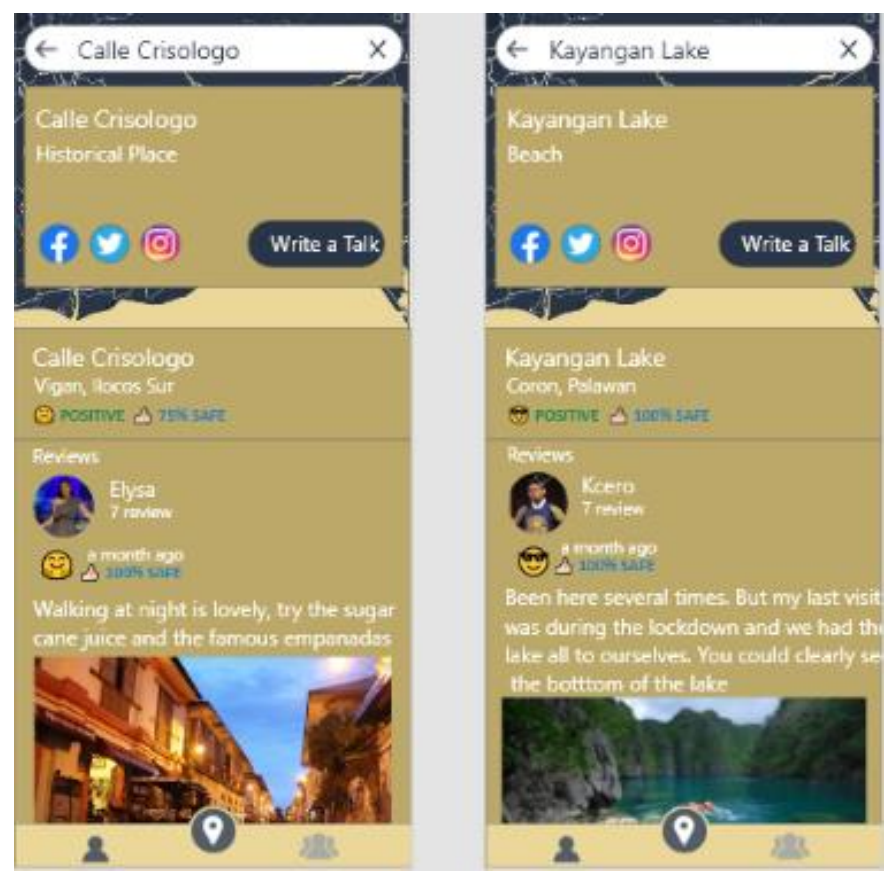

Fig. 5: User Feed

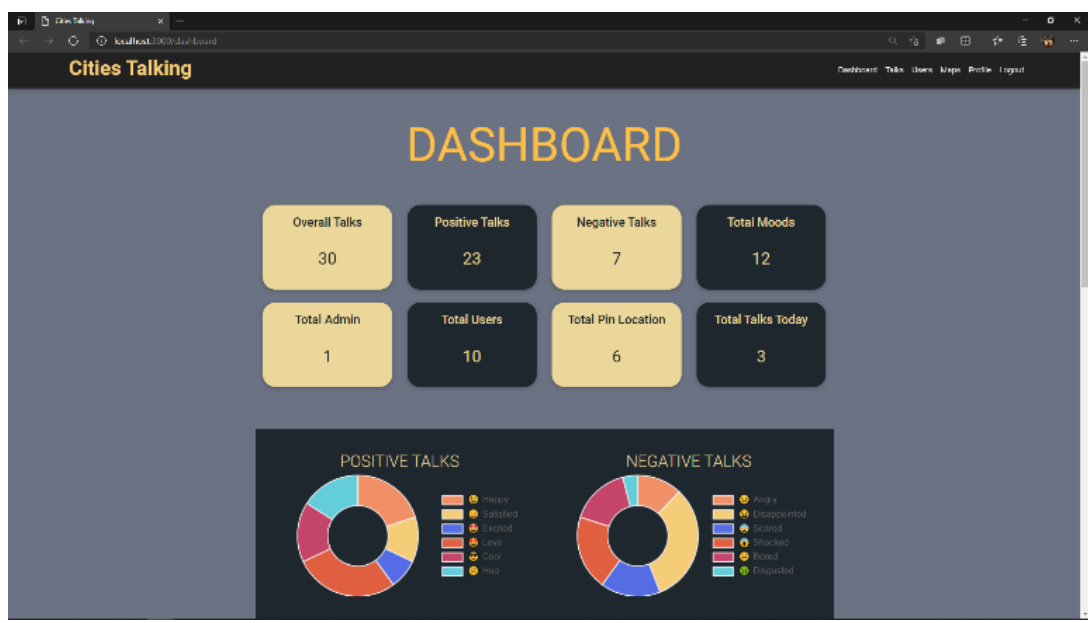

Fig. 6: Admin Dashboard

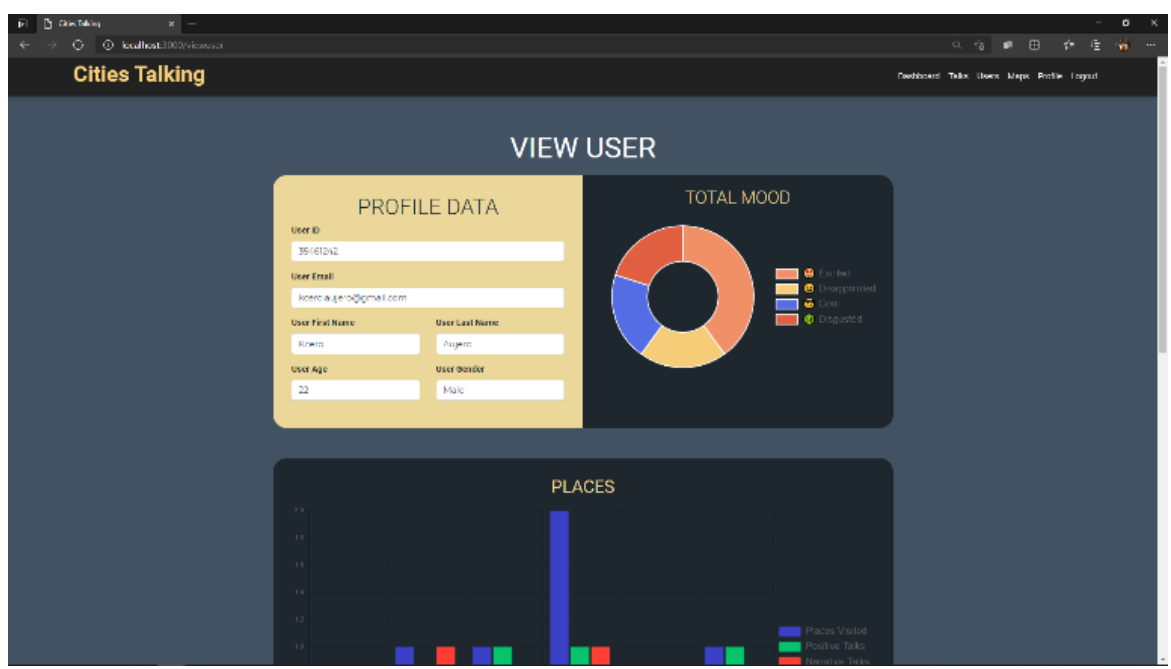

Fig. 7: View Users 


\section{Results and Discussion}

The results section summarizes the results obtained from the evaluation process. The proponents used the quantitative method approach by conducting online surveys for the end users along with the system. The developers selected a total of 10 respondents I.T. field as their background. The evaluation process of this project consists of 5 main factors: functionality, usability, reliability, performance, and supportability. The survey questionnaire given to the proponents followed the FURPS model in evaluating and rating the UI design as well as the functions of the system. With this, the proponents were given a set of questions in-line with the stated model and were prompted to answer using a Likert Scale where 1 means poor and 5 is Excellent. The purposive sampling method was also used to simplify the results gathered by the proponents.

Table 1: Summary of weighted mean

\begin{tabular}{|c|c|c|}
\hline Criteria & Weighted Mean & Response Description \\
\hline Functionality & 4.92 & Excellent \\
\hline Usability & 4.92 & Excellent \\
\hline Reliability & 4.8 & Excellent \\
\hline Performance & 4.93 & Excellent \\
\hline Sustainability & 4.95 & Excellent \\
\hline Overall & $\mathbf{4 . 9 0}$ & Excellent \\
\hline
\end{tabular}

Table 1 shows the summary of the weighted mean of the 5 criteria. Each criterion shows the weighted mean that had been gathered based on the score on the survey of the respondents. Based on the table above, Functionality scored a 4.92 which is equivalent to Excellent; Usability garnered a score of 4.92 belonging to the Excellent tier; Reliability fell short with 4.8 total weighted mean that corresponds with Excellent; Performance amounted to 4.93 which indicates Excellent; and lastly, Sustainability getting 4.95 weighted mean that signifies Excellent score. The total weighted mean of all the criteria amounts to 4.90 which classifies as an Excellent response.

\section{Conclusion}

The results section summarizes the results obtained from the evaluation process. The proponents used the quantitative method approach by conducting online surveys for the end users along with the system. The developers selected respondents in I.T. field as their background. The evaluation process of this project consists of 5 main factors: functionality, usability, reliability, performance, and supportability. The purposive sampling method was also used to simplify the results gathered by the proponents.

\section{Recommendation and Future Works}

Even with the success of the system, some limitations were evidently present as pointed out by users while using the system. To future researchers/developers, below are the gathered recommendation from this project's proponents that can improve the project and it contains three concrete recommendations: First, the Sentiment Analysis utilized in the system is only at entry level. It can only understand direct context of the English language. Plethora of words and phrases were not properly recognized either being by: Negation of Words, Sarcasm, Proverbs, Jargon Words, and Emojis, among others. Second, since the application is a crowd sourcing application, the users should be able to alert local authorities in case of emergencies. Using their GPS, they can easily pin-point the area of the emergency and fast respond can be accomplished. Lastly, users can only check-in and post pictures of the area, future developers can further improve user interactions by providing more features such as an e-commerce side or virtual games, among others.

\section{References}

[1] Evans, M. (2015). Importance of crowdsourcing. Retrieved from https://exinfm.com/board/crowdsourcing.htm

[2] Kyes, J. (2020, May 22). What is GPS? Retrieved from Geotab: https://www.geotab.com/blog/what-is-gps/

[3] How Has GPS Changed Our Life? (2020, August 25). Retrieved from Iconcox: 
https://www.iconcox.com/blog/how-has-gps-changed-our-

life.html\#: :text=It\%20has\%20changed\%20the\%20way,people\%20in\%20mapping\%20and\%20directions.

[4] Jonathan, C., \& Mokbel, M. (2018). Stella: geotagging images via crowdsourcing. Retrieved from ACM Digital Library.:https://doi.org/https://doi.org/10.1145/3274895.3274902

[5] Acampora, G., Anastasio, P., Risi, M., Tortora, G., \& Vitiello, A. (2020, July). Automatic Event Geo-Location in Twitter. Retrieved from https://www.researchgate.net/publication/342909180_Automatic_Event_GeoLocation_in_Twitter

[6] Bhaumik, U. (2020, November). Sentiment Analysis Using Twitter . Retrieved from https://www.researchgate.net/publication/346397445_Sentiment_Analysis_Using_Twitter

[7] Desai, A., Warner, J., Kuderer, N., Thompson, M., Painter, C., Lyman, G., \& Lopes, G. (2020, April 21). Crowdsourcing a crisis response for COVID-19 in oncology. Retrieved from https://www.nature.com/articles/s43018-020-0065-zKyes, J. (2020, May 22). What is GPS? Retrieved from Geotab: https://www.geotab.com/blog/what-is-gps/

[8] Gupta. (2018, January 8). Sentiment Analysis: Concept, Analysis, and Applications. Retrieved from Towards Data Science: https://towardsdatascience.com/sentiment-analysis-concept-analysis-and-applications-6c94d6f58c17 